Revue d'histoire de l'enfance « irrégulière »

Le Temps de l'histoire

15 | 2013

Enfances déplacées. (II) en temps de guerre

\title{
(lecture de) Allons enfants de la patrie. Génération Grande Guerre
}

Jean-Jacques Yvorel

\section{(2) OpenEdition \\ Journals}

Édition électronique

URL : http://journals.openedition.org/rhei/3540

DOI : $10.4000 /$ rhei.3540

ISSN : $1777-540 X$

Éditeur

Presses universitaires de Rennes

Édition imprimée

Date de publication : 30 octobre 2013

Pagination : 205-207

ISBN : 978-2-7535-2896-3

ISSN : $1287-2431$

Référence électronique

Jean-Jacques Yvorel, «(lecture de) Allons enfants de la patrie. Génération Grande Guerre », Revue d'histoire de l'enfance « irrégulière » [En ligne], 15 | 2013, mis en ligne le 30 octobre 2013, consulté le 10 décembre 2020. URL : http://journals.openedition.org/rhei/3540; DOI : https://doi.org/10.4000/rhei. 3540

Ce document a été généré automatiquement le 10 décembre 2020.

(c) PUR 


\title{
(lecture de) Allons enfants de la patrie. Génération Grande Guerre
}

\author{
Jean-Jacques Yvorel
}

\section{RÉFÉRENCE}

Paris, Seuil, collection « L'Univers historique », 438p. ISBN 9782021030822

Depuis le livre pionnier de Philippe Aries ${ }^{1}$, les travaux sur l'histoire de l'enfance se sont multipliés ${ }^{2}$. Le livre de Manon Pignot occupe une place particulière dans cette historiographie foisonnante. En effet, il ne s'agit pas ici d'analyser les discours et les représentations élaborés par les adultes sur l'enfance ou l'adolescence, encore moins de faire l'histoire des institutions destinées à l'enfance comme l'école, les colonies de vacances, les patronages ou les autres établissements éducatifs mais bien de faire une histoire de l'expérience enfantine. Pour faire cette histoire "à hauteur d'enfant », faire entendre "une parole enfantine et non plus seulement un discours sur l'enfance " (p.12), l'auteure a choisi une expérience forte et particulière, celle de la première guerre mondiale qui fournit «tout autant le sujet que les conditions d'un besoin d'expression personnelle » (p. 13).

Comment les enfants ont-ils réagi à cet événement pour eux à la fois subi et soudain que fut l'entrée en guerre? Manon Pignot essaie de répondre à cette question dans son premier chapitre intitulé "Rencontrer la guerre». Elle examine les réactions des enfants au départ des hommes, leurs pères souvent, et aux larmes des mères. Ceux qui fuient l'avance allemande témoignent de l'expérience de l'exode et ceux qui sont restés dans la zone occupée, du choc de l'invasion. Enfin, complétant en quelque sorte les travaux de Stéphane Audain-Rousseau sur la mobilisation culturelle et idéologique des enfants ${ }^{3}$, elle observe à partir des sources enfantines, le degré d'adhésion des garçons et des filles face à cette propagande. 
arrondissements pauvres de Paris notamment ne manquent pas d'évoquer, le froid, lui, touche tout le monde. La "Guerre de 14-18 " c'est plus d'un million d'orphelins en France, autant en Allemagne et 300000 en Grande-Bretagne. Le deuil est donc une expérience affective partagée par bien des enfants. Ils rencontrent la mort directement, quand ils perdent leur père mais aussi indirectement quand un camarade de classe perd le sien ou quand un adulte proche comme l'instituteur voit disparaître son fils. Les écoliers sont invités à imaginer la vie dans les tranchées et à la dessiner. Manon Pignot, qui a consacré un ouvrage aux dessins de guerre des enfants ${ }^{4}$, analyse ces « œuvres de commande " avec une grande finesse. Si les bombardements de la première guerre mondiale n'ont rien à voir en termes d'intensité, de dégâts et de nombre de victimes avec ceux de la seconde guerre mondiale, la terreur que suscitent les Zeppelins chez les enfants semble bien réelle. La présence des mutilés constitue une irruption de la guerre dans toute sa violence dans le quotidien des enfants. Quant à la rencontre effective avec les prisonniers allemands, elle amène des réflexions nouvelles sur l'humanité des abominables « boches».

4 «De l'arrière à la zone occupée [se dessine une] géographie de l'expérience de guerre enfantine » (chapitre III). Si, comme l'écrit l'auteure, « les expériences enfantines de la guerre reposent toutes sur une base commune, celle des mobilisations humaines et institutionnelles auxquelles les enfants ont été confrontés»(p.181), il n'y a pas homogénéité de la dite expérience. La variable géographique joue fortement avec, bien sûr, une profonde différence entre la zone occupée et la zone libre, sans qu'il y ait, pour autant, uniformité de l'arrière. Manon Pignot montre notamment qu'en zone libre la différenciation du vécu des enfants est marquée par des critères socio-économiques et par la distinction ville/campagne alors qu'en zone occupée le concept de genre est primordial car, ainsi que l'avait remarqué Annette Becker, «ce sont les femmes qui vont subir l'essentiel du fardeau de l'occupation ${ }^{5} »$. L'identification des petites filles et des adolescentes à leur mère est très forte et le caractère sexuel du rapport de soumission créé par la situation d'occupation n'échappe pas aux enfants.

5 La guerre a-t-elle permis "L'invention des pères" comme le suggère le titre du chapitre IV ? À tout le moins elle a donné une visibilité aux relations entre le père et ses enfants, en rendant obligatoire la médiation épistolaire. Privé du contact direct « tout le monde se met à écrire même les illettrés » (p. 269). Cette abondante correspondance dessine « une figure paternelle loin d'être monochrome, depuis l'héroïsation jusqu'à la destitution » (p. 270). Il est très difficile de résumer le kaléidoscope de sentiments mis à jour par Manon Pignot d'où il ressort cependant «un portrait des pères dont la caractéristique principale est l'expression nouvelle d'un sentiment paternel profond, nourri d'angoisses et chargé de puissants affects » (p. 332).

6 Dans un ultime chapitre intitulé «Sortir de la guerre, sortir de l'enfance » l'auteure montre "que la guerre n'est en rien une parenthèse pour les enfants qui la vivent » (p.333). La guerre et le deuil qui en découle et qui touche directement ou indirectement la quasi-totalité des enfants marquent durablement toute une génération à tel point que Manon Pignot n'hésite pas à écrire que «tout le champ sociopolitique de l'entre-deux-guerres pourrait être relu à l'aune de la question du deuil de guerre et de son impact» (p. 391). La restauration de la paix «n'induit pas pour autant de retour à la normale » (p. 393).

7 Au-delà des apports à la connaissance de la Grande Guerre, cet ouvrage est riche d'enseignements méthodologiques et propre à nourrir la réflexion de tous ceux qui 
travaillent sur l'histoire de l'enfance et de l'adolescence. Manon Pignot démontre qu'il est possible de retrouver, au moins pour partie, ce que sont la vision et le vécu de la guerre quand on a cinq, dix ou quinze ans. Journaux intimes, autobiographies, correspondances, rédactions scolaires, dessins et, quand on travaille sur des périodes récentes, entretiens avec les témoins, sont autant de sources qui permettent de redonner la parole à ceux qui sont souvent parlés mais qui ne parlent pas : les enfants et les adolescents. Cet ouvrage peut (doit?) inspirer les travaux sur l'enfance «irrégulière " même si le délinquant juvénile, le mineur maltraité, l'orphelin, l'inadapté, le blouson noir, la fille perdue recluse du Bon Pasteur... sont encore plus silencieux que les enfants "normaux» de Manon Pignot. Des travaux récents ont montré que là aussi des sources existent qui permettent de faire une histoire de l'expérience de "l'irrégularité » et de la « rééducation » du côté des adolescent(e)s. À côté des entretiens et des autobiographies ${ }^{6}$, on peut notamment utiliser les dossiers nominatifs tenus par les institutions ${ }^{7}$. Avec leurs lettres saisies, leurs dessins, leurs rédactions, leurs extraits d'entretiens retranscrits, ils viennent éclairer d'un jour nouveau « les ombres de l'histoire » en redonnant au moins partiellement la parole aux exclus et aux marginalisés.

\section{NOTES}

1. ARIES Philippe, L'enfant et la vie familiale sous l'Ancien Régime, Paris, Plon, 1960.

2. Citons deux ouvrages collectifs BECCHI Egle, JULIA Dominique, (dir.), Histoire de l'enfance en occident, Paris, seuil, 1994, 2 vol.; BARDET Jean-Pierre, LUC Jean-Noël, ROBIN-ROMERO Isabelle, ROLLET Catherine, (dir.), Lorsque l'enfant grandit. Entre dépendance et autonomie, Paris, Presses de l'Université de Paris-Sorbonne, 2003, 983 p.

3. AUDAIN-ROUSSEAU Stéphane, La guerre des enfants. 1914-1918. Essai d'histoire culturelle, Paris, Armand Colin, 1993, (rééd. 2004).

4. PIGNOT Manon, La guerre des crayons. Quand les petits parisiens dessinaient la Grande Guerre, Paris, Parigramme, 2004.

5. BECKERT ANNETTE, « Le sort des femmes pendant l'occupation allemande du nord de la France ", dans MORIN-ROTUREAU Évelyne (dir.), 1914-1918: combats de femmes. Les femmes pilier, de l'effort de guerre, Paris, Autrement, 2004, p. 153.

6. Nous avons fait une première recension des autobiographies « d'enfants de justice » imprimées dans "Autobiographies de ceux qui n'écrivent pas ", Les Cahiers dynamiques, $\mathrm{n}^{\circ} 46,2010$. Une exploration des fonds de l'Association pour l'autobiographie et le Patrimoine Autobiographique d'Ambérieu-en-Bugey permettrait sûrement d'enrichir cette liste.

7. Deux ouvrages collectifs ont été consacrés à ces dossiers dont nous avons rendu compte dans les colonnes de cette revue (Cf. $n^{\circ} 13$ ). Voir aussi bien sûr le $n^{\circ} 11$ de la RHEI dirigé par Mathias Gardet, Paroles libres, paroles captives. Lectures des dossiers de jeunes placés. 